\title{
THE DUST-UNBIASED COSMIC STAR-FORMATION HISTORY FROM THE 20 CM VLA-COSMOS SURVEY*
}

\author{
V. Smolčíc ${ }^{1,2,10}$, E. Schinnerer ${ }^{1}$, G. Zamorani ${ }^{3}$, E. F. Bell ${ }^{1}$, M. Bondi ${ }^{4}$, C. L. CARilli ${ }^{5}$, P. Ciliegi ${ }^{3}$, B. Mobasher ${ }^{6}$, \\ T. PAGLIONE ${ }^{7,8}$, M. SCODEGGIO ${ }^{9}$, AND N. SCOVILLE ${ }^{2}$ \\ ${ }^{1}$ Max Planck Institut für Astronomie, Königstuhl 17, Heidelberg, D-69117, Germany \\ 2 California Institute of Technology, MC 105-24, 1200 East California Boulevard, Pasadena, CA 91125, USA \\ ${ }^{3}$ Istituto Nazionale di Astrofisica, Osservatorio Astronomico di Bologna, via Ranzani 1, 40127, Bologna, Italy \\ ${ }^{4}$ Istituto Nazionale di Astrofisica, Istituto di Radioastronomia, via Gobetti 101, 40129 Bologna, Italy \\ 5 National Radio Astronomy Observatory, P.O. Box 0, Socorro, NM 87801-038, USA \\ ${ }^{6}$ Physics and Astronomy Department, University of California, Riverside, 900 University Ave, CA 92521, USA \\ 7 York College, City University of New York, 94-20 Guy R. Brewer Boulevard, Jamaica, NY 11451, USA \\ 8 American Museum of Natural History, Central Park West at 79th Street, New York, NY 10024, USA \\ 9 Istituto di Astrofisica Spaziale e Fisica Cosmica Milano-Istituto Nazionale di Astrofisica, Via Bassini 15, I-20133, Milan, Italy \\ Received 2008 April 24; accepted 2008 August 25; published 2008 December 1
}

\begin{abstract}
We derive the cosmic star-formation history out to $z=1.3$ using a sample of $\sim 350$ radio-selected star-forming (SF) galaxies, a far larger sample than those in previous, similar studies. We attempt to differentiate between radio emission from active galactic nuclei and SF galaxies, and determine an evolving $1.4 \mathrm{GHz}$ luminosity function (LF) based on these VLA-COSMOS SF galaxies. We precisely measure the high-luminosity end of the SF galaxy LF (star-formation rate $\gtrsim 100 M_{\odot} \mathrm{yr}^{-1}$; equivalent to ultra-luminous IR galaxies) out to $z=1.3$, finding a somewhat slower evolution than that previously derived from mid-infrared data. We find that more stars are forming in luminous starbursts at high redshift. We use extrapolations based on the local radio galaxy LF; assuming pure luminosity evolution, we derive $L_{*} \propto(1+z)^{2.1 \pm 0.2}$ or $L_{*} \propto(1+z)^{2.5 \pm 0.1}$, depending on the choice of the local radio galaxy LF. Thus, our radio-derived results independently confirm the $\sim 1$ order of magnitude decline in the CSFH since $z \sim 1$.
\end{abstract}

Key words: cosmology: observations - galaxies: evolution - galaxies: fundamental parameters - galaxies:

starburst - radio continuum: galaxies

Online-only material: color figure

\section{INTRODUCTION}

Studies based on different galaxy star-formation indicators (UV, optical, far-IR (FIR), radio) agree that the cosmic starformation history (CSFH, i.e., the total star-formation rate (SFR) per unit of co-moving volume) has declined by about an order of magnitude since $z \sim 1$ (for a compilation see e.g., Hopkins 2004; Hopkins \& Beacom 2006). One of the major difficulties of UV/optical based tracers is the significant model-dependent dust-obscuration correction that needs to be imposed on the data. This "dust-obscuration problem" may be overcome using longer wavelengths, such as the IR and radio regimes. However, in these cases a multiwavelength approach is essential as redshift information and a reliable identifier of star-forming (SF) galaxies is required (e.g., Caputi et al. 2007; Smolčić et al. 2008; S08 hereafter). In this context the radio star-formation tracer provides an important complementary view of the CSFH. First, radio emission is a dust-insensitive tracer of recent star formation (not affected by old stellar populations; see Condon 1992 for a review). Second, interferometric radio observations with $\sim 1^{\prime \prime}$ resolution allow more reliable identifications (compared with FIR and submilliter data) with objects detected at other wavelengths.

The dust-unbiased total CSFH has been constrained recently using mid-infrared (MIR) $(24 / 8 \mu \mathrm{m})$ selected samples obtained by deep small area surveys (CDFS, GEMS, GOODS;

\footnotetext{
* Based on observations with the National Radio Astronomy Observatory which is a facility of the National Science Foundation operated under cooperative agreement by Associated Universities, Inc.

${ }^{10}$ Fellow of the International Max Planck Research School for Astronomy and Cosmic Physics.
}

Le Floc'h et al. 2005; Zheng et al. 2006, 2007; Caputi et al. 2007; Bell et al. 2007). Small area surveys, however, are subject to cosmic variance. Moreover, they do not observe a largeenough comoving volume in order to fairly sample rare highluminosity galaxies. In this paper we use the $2 \mathrm{deg}^{2}$ COSMOS field (Scoville et al. 2007a), and its $1.4 \mathrm{GHz}$ radio observations (Schinnerer et al. 2007), to derive the CSFH. In such a large field cosmic variance is significantly reduced as $2 \mathrm{deg}^{2}$ $(1.4 \times 1.4)$ sample comoving volumes in the early universe $(z \sim 1)$ comparable with the largest survey in the local universe (Sloan Digital Sky Survey Data Release 1 (SDSS-DR1); see Figure 1 in Scoville et al. 2007a). The physical angular size sampled by 1.4 at redshifts of $0.2-1.1$ roughly corresponds to a factor of 3-8 of the typical cluster scale length $(\sim 5 \mathrm{Mpc})$. Thus at all redshifts, such a field fairly samples relevant structures in the universe (see Scoville et al. 2007a, 2007b, McCracken et al. 2007 for a more detailed discussion on cosmic variance in the COSMOS field).

In the last decade several radio surveys have been utilized to independently derive the CSFH. However, their results are based on small observed areas, nonuniform rms in the final map, as well as a fairly nonuniform selection of SF galaxies. The first derivation of the CSFH based on radio data has been performed by Haarsma et al. (2000). They combined three radio frequency observations of the Hubble Deep Field (66 arcmin'; Richards et al. 1998), SSA13 (7 $\operatorname{arcmin}^{2}$; Windhorst et al. 1995), and V15 (86 $\operatorname{arcmin}^{2}$; Fomalont et al. 1991; Hammer et al. 1995) fields reaching $5 \sigma$ radio depths of $9,8.8$, and $16 \mu \mathrm{Jy}$ at the field centers, respectively. Of the total number of their radio-selected sources (77) only 37 were securely classified as SF galaxies (based on morphology and/or optical spectroscopy). Their 
sources reach out to $z \sim 3,23$ have spectroscopic redshifts, and the redshifts for the remaining 14 have been estimated using only $I$ or $H$ and $K^{\prime}$ bands (see Haarsma et al. 2000 for details). More recently, Seymour et al. (2008) used radio observations of the $13^{\mathrm{H}}$ XMM-Newton/Chandra Deep field (0.196 $\mathrm{deg}^{2}$; $4 \sigma \sim 30 \mu \mathrm{Jy})$ to derive the CSFH. Out of a total of 449 radio sources they find 269 galaxies which they classify as SF based on a number of criteria applicable to subsamples of their objects (see their Table 1). About half of these galaxies have a spectroscopic redshift $(z \lesssim 3)$.

Here we utilize the $1.4 \mathrm{GHz}$ Very Large Array (VLA) ${ }^{11}$ observations of the COSMOS $2 \mathrm{deg}^{2}$ field (VLA-COSMOS Large Project; Schinnerer et al. 2007) to overcome the abovementioned biases. The final mosaic has a resolution of $1^{\prime \prime} .5 \times 11^{\prime \prime} .4$ and a typical rms of $\sim 10.5(15) \mu \mathrm{Jy}_{\text {beam }}{ }^{-1}$ in the central 1 (2) $\mathrm{deg}^{2}$ making this survey to date the largest radio deep field at this sensitivity and angular resolution. Given the COSMOS panchromatic data set, S08 have developed a novel method to select SF and active galactic nucleus (AGN) galaxies based only on NUV-near-IR (NIR) photometry. This yielded a robust statistical selection of $340 \mathrm{SF}$ galaxies out to $z=1.3$ (see Section 2.1). One particular advantage of the VLA-COSMOS survey is the large comoving volumes observed at all redshifts, thus allowing one to study a statistically significant sample of rare objects, such as the most intensely SF galaxies. In this paper we focus on the derivation of the CSFH, with emphasis on the evolution of galaxies with high SFRs $\left(\gtrsim 100 M_{\odot} \mathrm{yr}^{-1}\right)$, using the $1.4 \mathrm{GHz}$ VLA-COSMOS Large Project.

We report magnitudes in the $\mathrm{AB}$ system, adopt a standard concordance cosmology $\left(H_{0}=70, \Omega_{M}=0.3, \Omega_{\Lambda}=0.7\right)$, and define the radio synchrotron spectrum as $F_{v} \propto v^{-\alpha}$, assuming $\alpha=0.7$.

\section{THE $1.4 \mathrm{GHz}$ LUMINOSITY FUNCTION FOR STAR-FORMING GALAXIES IN VLA-COSMOS}

\subsection{The Star-Forming Galaxy Sample}

The sample of SF galaxies used here is presented in S08, and briefly summarized below. Using radio and optical data for the COSMOS field S08 have constructed a sample of 340 SF galaxies with $z \leqslant 1.3$ out of the entire VLA-COSMOS catalog. The selection required optical counterparts with $i \leqslant$ 26 , accurate photometry (i.e., outside photometrically flagged areas), and an signal-to-noise ratio $(\mathrm{S} / \mathrm{N}) \geqslant 5$ detection at $20 \mathrm{~cm}$, and is based on rest-frame optical colors (see also Smolčić et al. 2006). The classification method was well calibrated using a large local sample of galaxies $(\sim 7,000$ SDSS "main" spectroscopic galaxy sample, NRAO VLA Sky Survey (NVSS) and IRAS surveys) representative of the VLACOSMOS population. It was shown that the method agrees remarkably well with other independent classification schemes based on MIR colors and optical spectroscopy, and that, within the selection limits, it is not biased against dusty starburst galaxies. It is important to note that the rest-frame colors for the VLA-COSMOS radio sources have been shown to stay basically unchanged with redshift (an effect that was also noted by Barger et al. 2007 and Huynh et al. 2008).

Out of the 340 selected SF galaxies 150 have spectroscopic redshifts available, while the remaining sources have very

\footnotetext{
11 VLA is operated by the National Radio Astronomy Observatory (NRAO), which is a facility of the National Science Foundation, operated under cooperative agreement by Associated Universities, Inc.
}

reliable photometric redshifts (see S08 and references therein). Based on Monte Carlo simulations, S08 have shown that the photometric errors in the rest-frame color introduce a small, $\sim 5 \%$, number incompleteness of SF galaxies. Here we use the S08 sample of SF galaxies, statistically corrected for this effect.

\subsection{Derivation of the Radio Luminosity Function (LF)}

We derive the radio luminosity function $(L F ; \Phi)$ for our $\mathrm{SF}$ galaxies in four redshift bins using the standard $1 / V_{\max }$ method (Schmidt 1968). In computing $V_{\max }$ we take into account both the radio and optical flux limits (i.e., the maximum observable redshift, $z_{\max }$ ), as well as the nonuniform rms noise level in the VLA-COSMOS mosaic. For the latter we use the differential visibility area (i.e., areal coverage, $A_{k}$, versus rms; see Figure 13 in Schinnerer et al. 2007). For a source with $1.4 \mathrm{GHz}$ luminosity $L_{j}$, its maximum volume is then $V_{\max }\left(L_{j}\right)=$ $\sum_{k=1}^{n} A_{k} \cdot V_{\max }\left(z_{\max }^{A_{k}}, L_{j}\right)$.

Several additional corrections need to be taken into account: (1) the VLA-COSMOS detection completeness, (2) the fraction of sources not included in the radio-optical sample, and (3) the SF galaxy selection bias due to the rest-frame color uncertainties.

The radio detection completeness of the VLA-COSMOS survey has been derived by Bondi et al. (2008) via Monte Carlo-artificial source-simulations for the inner $1 \mathrm{deg}^{2}$. Artificial radio sources have been simulated taking into account both the flux density and angular size distributions. The first has been described using a typical broken power law, while the latter has been assumed to depend on the flux density $\left(\Theta_{\text {med }} \propto F_{v}^{m}\right)^{12}$ The median of these corrections is $\sim 10 \%$ (reaching a maximum value of $60 \%$ in only one of the lowest-flux-density bins; see Table 1 in Bondi et al. 2008). Assuming that these corrections, scaled for the differences in radio sensitivity, hold for the full $2 \mathrm{deg}^{2}$, we utilize them to correct our LFs for the detection incompleteness $\left(f_{\text {det }}\right)$.

To correct for objects located in photometrically flagged regions in the optical images, or not identified with an optical counterpart (and thus not included in the radio-optical sample), we construct a correction curve as a function of total $1.4 \mathrm{GHz}$ flux density which yields an average correction of $\sim 30 \%$ (see Figure 23 in S08; $f_{\text {flag }}$ ). Hence, in the $i^{\text {th }}$ luminosity bin, the comoving space density $\left(\Phi_{i}\right)$ and its corresponding error $\left(\sigma_{i}\right)$ are computed by weighing the contribution of each galaxy by the two correction factors, $f_{\text {det }}$ and $f_{\text {flag }}$, which were obtained by linearly interpolating the two correction curves described above, respectively, at the total flux of the given, $j^{\text {th }}$, source:

$$
\Phi_{i}=\sum_{j=1}^{N} \frac{f_{\mathrm{det}}^{j} \cdot f_{\mathrm{flag}}^{j}}{V_{\max }^{j}} ; \quad \sigma_{i}=\sqrt{\sum_{j=1}^{N}\left(\frac{f_{\mathrm{det}}^{j} \cdot f_{\mathrm{flag}}^{j}}{V_{\max }^{j}}\right)^{2}} .
$$

\footnotetext{
12 The angular-size dependence of radio sources on their flux density has been observed in numerous radio surveys, and it is mainly due to the radio luminosity-size relation. At fainter flux densities, intrinsically fainter (and therefore smaller) radio sources are preferentially sampled. Comparisons between the median angular sizes in different flux ranges and between real and simulated $1.4 \mathrm{GHz}$ radio data showed that the median angular size $\left(\Theta_{\mathrm{med}}\right)$ of radio sources changes with flux according to a power law with an exponent of $m=0.5 ; \Theta_{\text {med }} \propto F_{v}^{0.5}$ yields flux-dependent completeness corrections.
} 
Table 1

1.4 GHz Radio Luminosity Functions for VLA-COSMOS Star-Forming Galaxies $\left(H_{0}=70, \Omega_{M}=0.3, \Omega_{\Lambda}=0.7\right)$

\begin{tabular}{|c|c|c|}
\hline $\begin{array}{l}\text { Redshift } \\
\text { Range }\end{array}$ & $\begin{array}{c}L_{1.4 \mathrm{GHz}} \\
\left(\mathrm{W} \mathrm{Hz}^{-1}\right)\end{array}$ & $\begin{array}{c}\Phi \\
\left(\mathrm{Mpc}^{-3} \mathrm{dex}^{-1}\right)\end{array}$ \\
\hline \multirow{7}{*}{$0.1<z \leqslant 0.35$} & $1.0 \times 10^{22}$ & $7.0 \pm 1.5 \times 10^{-4}$ \\
\hline & $2.0 \times 10^{22}$ & $2.9 \pm 0.7 \times 10^{-4}$ \\
\hline & $4.0 \times 10^{22}$ & $3.5 \pm 0.6 \times 10^{-4}$ \\
\hline & $8.9 \times 10^{22}$ & $5.0 \pm 2.1 \times 10^{-5}$ \\
\hline & $1.8 \times 10^{23}$ & $3.2 \pm 1.6 \times 10^{-5}$ \\
\hline & $3.2 \times 10^{23}$ & $1.4 \pm 1.0 \times 10^{-5}$ \\
\hline & $3.5 \times 10^{22}$ & $2.4 \pm 0.9 \times 10^{-4}$ \\
\hline \multirow[t]{6}{*}{$0.35<z \leqslant 0.6$} & $6.3 \times 10^{22}$ & $1.5 \pm 0.4 \times 10^{-4}$ \\
\hline & $1.0 \times 10^{23}$ & $1.3 \pm 0.3 \times 10^{-4}$ \\
\hline & $1.6 \times 10^{23}$ & $5.8 \pm 1.6 \times 10^{-5}$ \\
\hline & $2.2 \times 10^{23}$ & $9.0 \pm 6.4 \times 10^{-6}$ \\
\hline & $3.5 \times 10^{23}$ & $1.5 \pm 0.7 \times 10^{-6}$ \\
\hline & $1.4 \times 10^{23}$ & $1.2 \pm 0.2 \times 10^{-5}$ \\
\hline \multirow[t]{6}{*}{$0.6<z \leqslant 0.9$} & $2.8 \times 10^{23}$ & $4.8 \pm 0.9 \times 10^{-5}$ \\
\hline & $5.6 \times 10^{23}$ & $1.7 \pm 0.5 \times 10^{-5}$ \\
\hline & $1.1 \times 10^{24}$ & $5.8 \pm 2.6 \times 10^{-6}$ \\
\hline & $2.5 \times 10^{24}$ & $5.2 \pm 2.3 \times 10^{-6}$ \\
\hline & $5.0 \times 10^{24}$ & $2.0 \pm 1.4 \times 10^{-6}$ \\
\hline & $3.2 \times 10^{23}$ & $9.2 \pm 1.5 \times 10^{-5}$ \\
\hline \multirow[t]{6}{*}{$0.9<z \leqslant 1.3$} & $6.3 \times 10^{23}$ & $2.4 \pm 0.5 \times 10^{-5}$ \\
\hline & $1.3 \times 10^{24}$ & $1.8 \pm 0.4 \times 10^{-5}$ \\
\hline & $2.8 \times 10^{24}$ & $3.2 \pm 1.4 \times 10^{-6}$ \\
\hline & $5.6 \times 10^{24}$ & $1.2 \pm 0.8 \times 10^{-6}$ \\
\hline & $1.0 \times 10^{25}$ & $2.0 \pm 1.0 \times 10^{-6}$ \\
\hline & $1.8 \times 10^{25}$ & $1.0 \pm 0.7 \times 10^{-6}$ \\
\hline
\end{tabular}

The selection bias due to the rest-frame color uncertainties is accounted for via Monte Carlo simulations. As described in S08, the rest-frame color error distribution is simulated using a Gaussian function with $\sigma=0.1$. These errors are then added to the rest-frame color obtained from the spectral energy distribution (SED) fitting. The SF galaxies are then selected, and the LF is derived as described above. This process is repeated 300 times; hence we obtain 300 realizations of $\left(\Phi_{i}, \sigma_{i}\right)$ for each luminosity bin. We take the median values as representative.

\subsection{The Radio Luminosity Function}

The LFs for our SF galaxies for the four chosen redshift bins are shown in Figure 1, and tabulated in Table 1. In each panel in Figure 1 we show the analytical form of the locally-derived $20 \mathrm{~cm}$ LF for SF galaxies given by Sadler et al. (2002, see also Section 2.4). It is worth noting that although the $2 \mathrm{deg}^{2}$ COSMOS field samples a relatively small comoving volume at the lowest redshifts and only a photometric identification of SF galaxies has been used, our LF in the lowest-redshift bin agrees remarkably well with the local LFs that were derived using allsky radio surveys (NVSS) combined with good-quality optical spectroscopic data (SDSS, Two-Degree Field) to identify SF galaxies. For comparison, in the first redshift bin we also indicate other local radio LFs that exist in the literature (Condon 1989; Best et al. 2005). We discuss the implications of the differences between the various local radio LFs on the star-formation history in Section 2.4.1.

In all panels we show the volume densities of $20 \mathrm{~cm}$ radio sources derived by Haarsma et al. (2000, gray asterisk), corrected to the current cosmology. Haarsma et al. used $37 \mathrm{SF}$ galaxies to derive these LFs $38 \%$ of these had approximate redshifts derived from $I$ - or $H K^{\prime}$-band magnitudes, the others

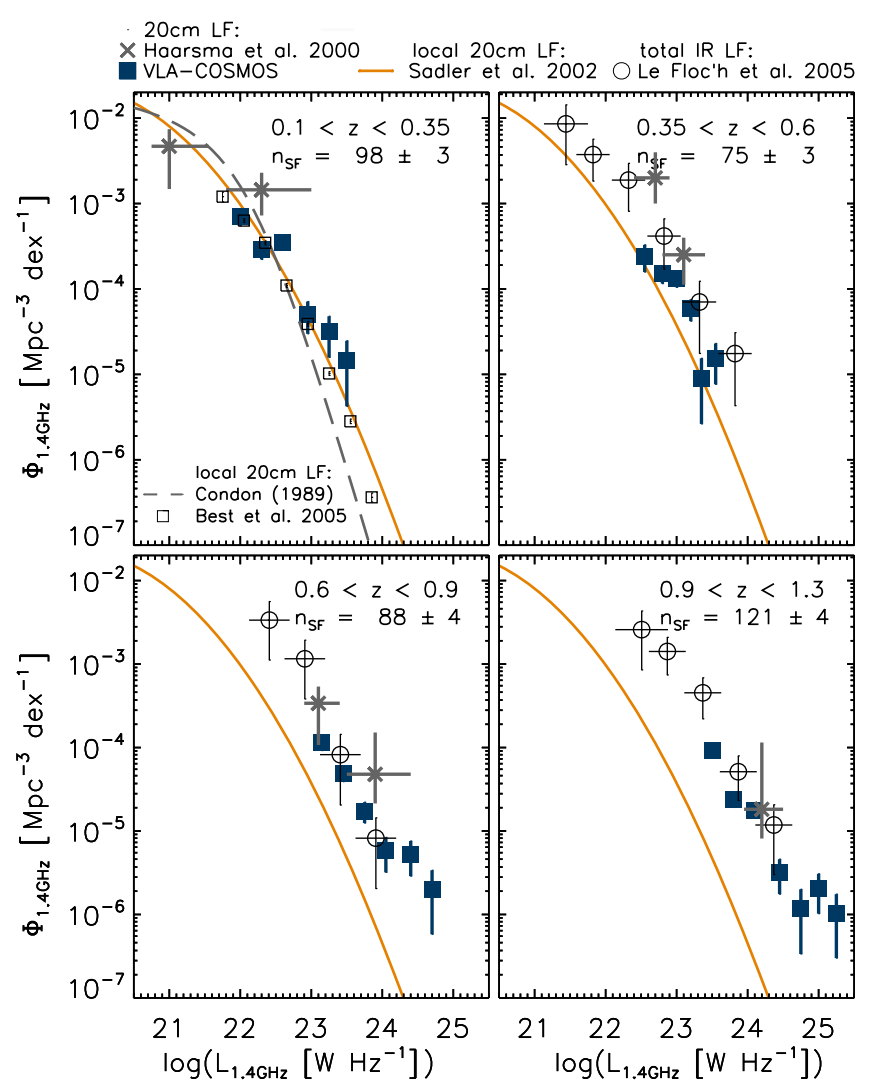

Figure 1. 1.4 GHz LFs for SF galaxies in the VLA-COSMOS survey, shown for four redshift ranges (filled blue squares), are presented in each panel. The number of galaxies in each redshift bin, statistically corrected for selection uncertainties (see the text for details), is also indicated in each panel. The local $20 \mathrm{~cm} \mathrm{LFs} \mathrm{for} \mathrm{SF} \mathrm{galaxies} \mathrm{are} \mathrm{shown} \mathrm{in} \mathrm{the} \mathrm{top-left} \mathrm{panel} \mathrm{(Condon} \mathrm{1989;} \mathrm{Sadler}$ et al. 2002; Best et al. 2005; the Sadler et al. 2002 LF, indicated by a solid orange curve, is shown in all panels). In all panels we show the volume densities derived by Haarsma et al. (2000; gray asterisk), corrected to the current cosmology. In the top right, and bottom panels, the total IR LFs (Le Floc'h et al. 2005) for the corresponding redshift ranges (open circles) is also shown (see the text for details). The total IR luminosity was converted to $1.4 \mathrm{GHz}$ luminosity using the correlation given in Bell (2003; their Equation (1)).

had spectroscopic redshifts). Their data points in each redshift range agree fairly well, within the error bars, with our results. Note that due to the almost one order of magnitude larger sample of SF galaxies used here, the error bars of the VLA-COSMOS LFs are significantly smaller.

In the higher-redshift panels we also compare our LFs with the total IR LFs derived by Le Floc'h et al. (2005, hereafter LF05) based on a $24 \mu \mathrm{m}$ selected sample in the Chandra Deep Field South (CDF-S; top right and both bottom panels in Figure 1). The total IR luminosity was converted to $1.4 \mathrm{GHz}$ luminosity using the total IR-radio correlation (Bell 2003), which has an intrinsic scatter of $\sim 0.26$ dex, shown by horizontal error bars in Figure 1. The IR LFs were rescaled to our redshift ranges either by combining two narrower redshift bins given in LF05 or by scaling a given comoving density using the evolution parameters and their corresponding errors, which are given in LF05. There is an excellent agreement between the $1.4 \mathrm{GHz}$ and IR LFs. Note also that the VLA-COSMOS LFs constrain the high-luminosity end much better than the IR data, i.e., the most intensely SF galaxies. Interestingly, the VLA-COSMOS SF galaxies in our two highest-redshift bins $(z>0.6)$ show an extended high-luminosity $\left(L_{1.4} \gtrsim 2 \times 10^{24} \mathrm{~W} \mathrm{~Hz}^{-1}\right)$ tail. We cannot exclude some possible contamination from AGNs, which are much more numerous than SF galaxies at these high radio 
luminosities (see Figure 17 in S08). However, a similar excess of SF galaxy volume densities at the high-luminosity end has been recently found by Cowie et al. (2004), who have used the Hubble Deep Field-North (HDF-N) and Small Science Aperture (SSA) 13 fields to derive the radio LF for spectroscopically identified SF galaxies (106 SF galaxies, $z \leqslant 1.4$ ).

\subsection{Toward the Derivation of the Cosmic Star-Formation History}

The comparison of our derived LFs (see Figure 1) with the local $20 \mathrm{~cm}$ LF shows a strong evolution with lookback time. The evolution of a given population of objects is usually parameterized with monotonic density and luminosity evolution. However, as the VLA-COSMOS data do not allow the derivation of the LF out to, and fainter than the characteristic luminosity (i.e., the "knee" of the LF), a full determination of the evolution is not possible with these data, and we choose to parameterize it using pure luminosity evolution. However, as we show below, its quantization depends fairly strongly on the choice of the local LF, as those presented in the literature are not exactly the same.

\subsubsection{The Local $20 \mathrm{~cm}$ Luminosity Functions}

The two commonly used analytical forms for the local $20 \mathrm{~cm}$ radio LFs have been presented in Condon (1989) and Sadler et al. (2002). Condon (1989) uses a hyperbolic parameterization (see also Condon et al. 2002) of the form:

$$
\begin{aligned}
\log \Phi_{1.4 \mathrm{GHz}}= & 28.83-\frac{3}{2} \log L_{1.4 \mathrm{GHz}}+Y \\
& -\left[B^{2}+\frac{\left(\log L_{1.4 \mathrm{GHz}}-X\right)^{2}}{W^{2}}\right]^{1 / 2},
\end{aligned}
$$

where $Y=2.88, B=1.5, X=22.108, W=0.667$ (corrected to the current cosmology and the base of $d \log L$, compared with $d$ mag given in Condon 1989). These parameters have been derived using a sample of 307 spiral and irregular galaxies from the Revised Shapley-Ames Catalog (RSA; Sandage et al. 1981) observed at $1.49 \mathrm{GHz}$ (Condon 1987, see Figure 2).

The LF given in Sadler et al. (2002) takes on the form of a combined power-law and Gaussian distribution given by the following analytic function (first proposed by Sandage et al. 1979):

$$
\Phi(L)=\Phi^{*}\left[\frac{L}{L_{*}}\right]^{1-\alpha} \exp \left\{-\frac{1}{2 \sigma^{2}}\left[\log \left(1+\frac{L}{L_{*}}\right)\right]^{2}\right\},
$$

with $\alpha=0.84, \sigma=0.94, \Phi^{*}=22.9 \times 10^{-3} \mathrm{Mpc}^{-3}$, and $L^{*}=1.95 \times 10^{19} \mathrm{~W} \mathrm{~Hz}^{-1}$ (scaled to the cosmology used here, and to the base of $d \log L$; see Hopkins 2004). Sadler et al. (2002) have used 204 SF galaxies drawn from the Two-Degree Field Galaxy Redshift Survey (2dFGRS) and the 1.4 GHz NVSS to derive their LF (see Figure 2). However, to obtain the bestfit parameters to Equation (3), they combined this sample (that constrains well the high-luminosity end of the LF; see Figure 2) with the RSA galaxy sample (in order to sample the lowluminosity end of the LF).

The differences between the two local LFs are illustrated in Figure 2. There is a discrepancy between the two analytical representations of the radio LFs at both the high- and low-luminosity ends. However, the discrepancy most severely affecting the star-formation rate density (SFRD), which we aim

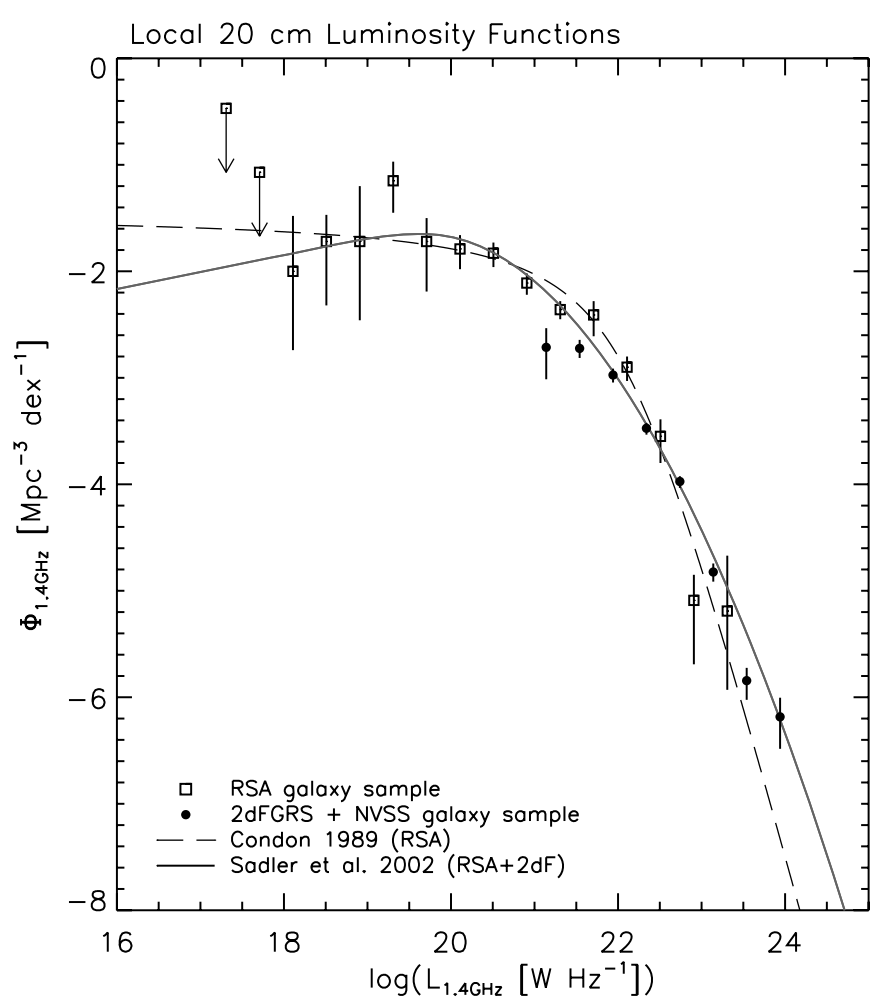

Figure 2. An illustration of the differences in the shapes of the local 1.4 GHz radio LFs found in the literature (Condon 1989, Sadler et al. 2002, corrected to the current cosmology). Also shown are the data points used for the analytical fits in Condon (1989, RSA) and Sadler et al. (2002, NVSS/2dFGRS and RSA samples). Note that the major difference in the representation of the local radio LFs is the analytical form used to fit the data (see Equations (2) and (3)), which particularly affect the position of the LF break, i.e., "knee".

to derive here, is the different position of the LF's turnover point $\left(L_{*}\right)$ given by the two analytic forms. This yields a difference of $10-50 \%$ in the SFRD integral (see e.g., Figure 3 and Figure 5) as the luminosity range encompassing the turnover point $\left(10^{19} \mathrm{~W} \mathrm{~Hz}^{-1} \lesssim L_{1.4 \mathrm{GHz}} \lesssim 10^{23} \mathrm{~W} \mathrm{~Hz}^{-1}\right)$ contributes the most $(\sim 95 \%)$ to the integral. It is important to note that the 2dFGRS and NVSS data used by the Sadler et al. (2002) sample the high-luminosity end more precisely (see Figure 2) of the local radio LF when compared with the RSA sample (Condon 1989). Hence, in Section 3.2 we will use the former to constrain the evolution of our most intensely SF galaxies (i.e., $L_{1.4} \gtrsim 2.34 \times 10^{23} \mathrm{~W} \mathrm{~Hz}^{-1}$ ). As the total SFRD in each redshift range is derived by integrating under the evolved LF curve (multiplied with the SFR; see below) down to the faintest $1.4 \mathrm{GHz}$ luminosities, we will take this difference of the local $20 \mathrm{~cm}$ LFs into account in further analysis.

\subsection{The Evolution of Star-Forming Galaxies}

We parameterize the evolution of the VLA-COSMOS SF galaxy LF by pure luminosity evolution:

$$
\Phi_{z}(L)=\Phi_{z=0}\left[\frac{L}{(1+z)^{\alpha_{L}}}\right],
$$

where $\alpha_{L}$ is the characteristic luminosity evolution parameter, and $\Phi_{z}$ is the LF at redshift $z$. We derive the evolution by summing the $\chi^{2}$ distributions obtained for a large range of fixed $\alpha_{L}$ in each redshift bin (excluding our first-localredshift bin). The uncertainty in $\alpha_{L}$ is then taken to be the 


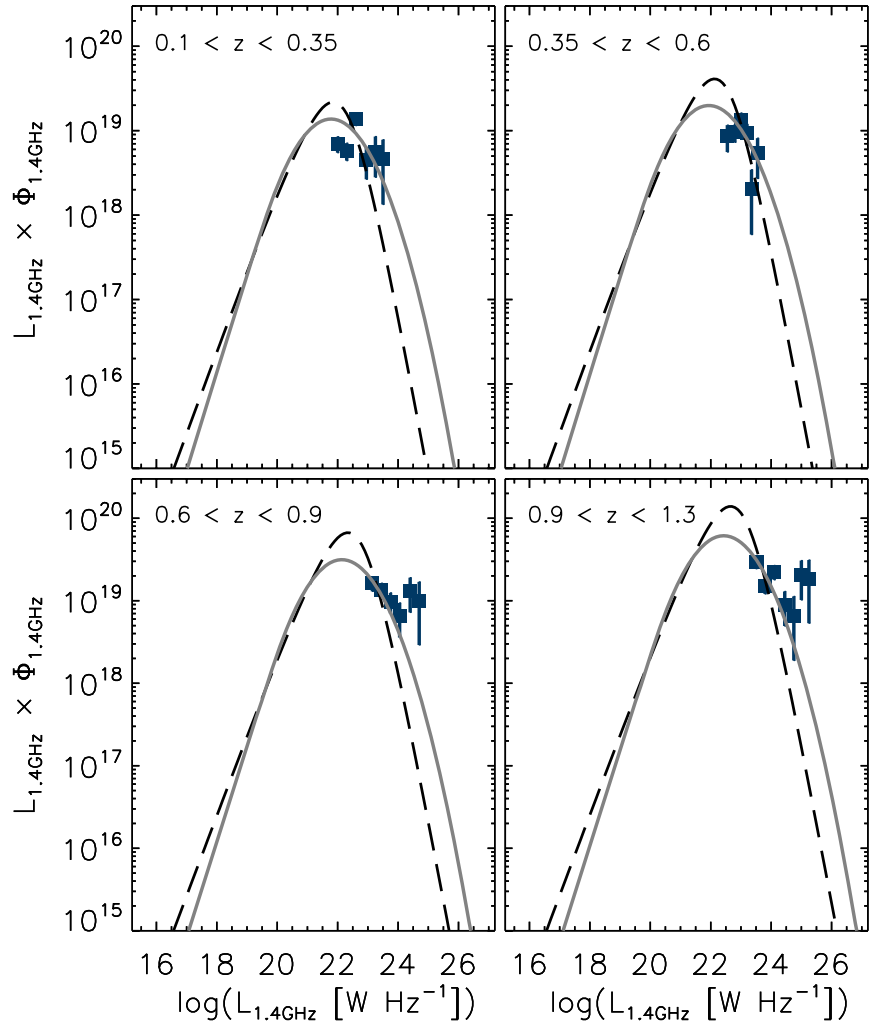

Figure 3. Luminosity density for VLA-COSMOS SF galaxies (filled squares) in four redshift bins. The solid and dashed curves correspond to the best-fit LFs in each redshift bin using the local LF given in Sadler et al. (2002) and Condon (1989), respectively. Note that the first appears to describe the bright-luminosity end more consistently with our data.

(A color version of this figure is available in the online journal.)

$2 \sigma$ error obtained from the $\chi^{2}$ statistics. Our results yield a pure luminosity evolution with (1) $\alpha_{L}=2.1 \pm 0.2$, when the Sadler et al. (2002) local LF is used, and (2) $\alpha_{L}=2.5 \pm 0.1$ when the Condon (1989) local LF is used. The different evolution parameters are a natural consequence of the different slopes of the two local LFs in the luminosity range that is constrained by the VLA-COSMOS data (see Figures 2 and 3).

Haarsma et al. (2000) have found that a pure luminosity evolution with $\alpha_{L} \approx 2.74$ is a good representation of the evolution of their radio-selected SF galaxies (no uncertainties were associated with this estimate). They used the Condon (1989) local LF as the basis for deriving their evolution. Given (1) their much smaller sample size, and (2) that their LF, when corrected for cosmology, agrees well with the one derived here (see Figure 1), these two results are in good agreement. Further, Cowie et al. (2004) find an evolution of the SF galaxy LF (using the Condon 1989 local LF) consistent with $\alpha_{L}$ of 3. The SF LFs, as well as the evolution, derived here agree with their findings (see Figure 3 in Cowie et al. 2004). Our results are also consistent with the overall evolution of SF galaxies obtained by Hopkins (2004, $\alpha_{L}=2.7 \pm 0.6$ ), that has been shown to fit well the evolution of radio-selected SF galaxies at low redshifts $(z \leqslant 0.5$; Afonso et al. 2005, Phoenix Deep Survey).

\section{THE COSMIC STAR-FORMATION HISTORY (CSFH)}

\subsection{The Total Cosmic Star-Formation History}

As the SFRD is derived by integrating the luminosity density, in Figure 3 we show the luminosity density for our four redshift

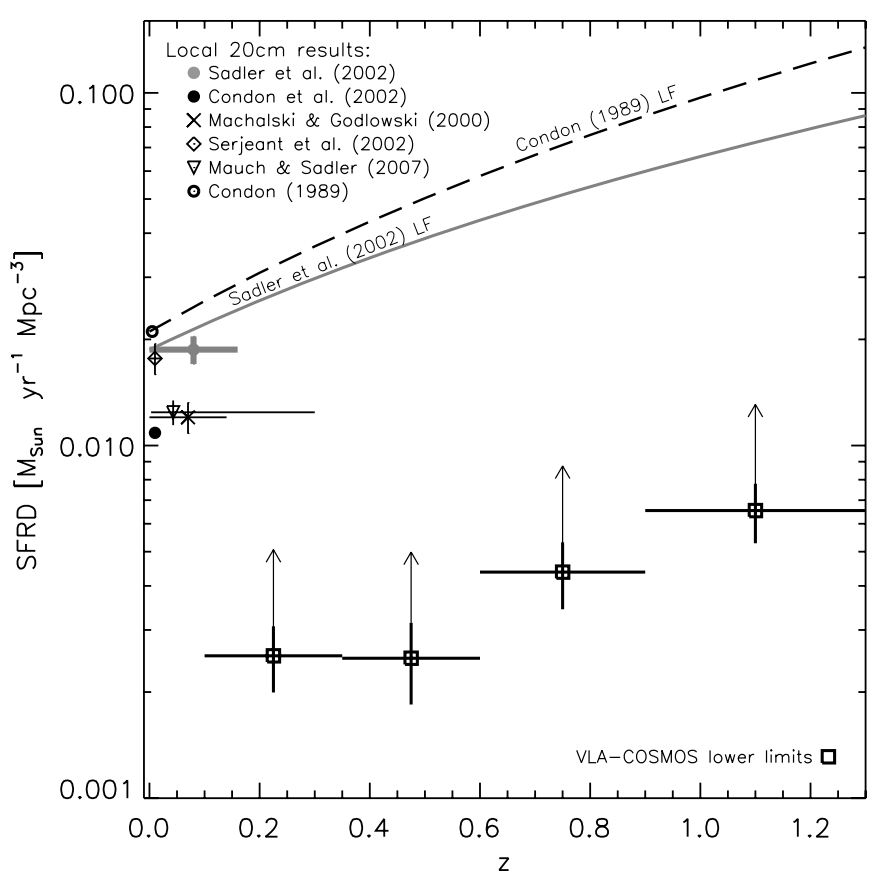

Figure 4. SFRD as a function of redshift. The open squares represent the SFRD derived from pure VLA-COSMOS data, without any extrapolations toward the faint or bright luminosity end. Therefore, they present strict lower limits, and are indicated with arrows. The two curves shown correspond to the average evolution of the VLA-COSMOS SF galaxies derived using two different local radio LFs (Condon 1989; Sadler et al. 2002). Local SFRDs derived by various authors are also shown, and are indicated in the panel.

bins. The shown curves are the two local $20 \mathrm{~cm}$ LFs (Condon 1989; Sadler et al. 2002), purely evolved in luminosity, and best fit to the VLA-COSMOS data in each redshift range. Prior to integration, we convert the $1.4 \mathrm{GHz}$ radio luminosity to SFRs ( $\psi$, in $M_{\odot} \mathrm{yr}^{-1}$ ), using the calibration given in Bell (2003), based on the total IR-radio correlation:

$\psi\left[M_{\odot} \mathrm{yr}^{-1}\right]= \begin{cases}5.52 \times 10^{-22} L_{1.4 \mathrm{GHz}}, & L_{1.4 \mathrm{GHz}}>L_{\mathrm{c}} \\ \frac{5.52 \times 10^{-22}}{0.1+0.9\left(L_{1.4 \mathrm{GH} z} / L_{c}\right)^{0.3}} L_{1.4 \mathrm{GHz}}, & L_{1.4 \mathrm{GHz}} \leqslant L_{\mathrm{c}},\end{cases}$

where $L_{\mathrm{c}}=6.4 \times 10^{21} \mathrm{~W} \mathrm{~Hz}^{-1}$, and $L_{1.4 \mathrm{GHz}}$ is the $1.4 \mathrm{GHz}$ radio luminosity in units of $\mathrm{W} \mathrm{Hz}^{-1}$. This calibration uses a Salpeter initial mass function (IMF $\propto M^{-2.35}$ ) from 0.1-100 $M_{\odot}$. After the conversion, we compute the SFRD for a given redshift bin as $\int \psi(L) \Phi_{z}(L) d L$, where $\Phi_{z}$ is the evolved radio LF that is best fitted to the data in each redshift range (see curves in Figure 3).

In Figure 4 we first show the SFRD, obtained by numerically integrating the VLA-COSMOS data (open squares) within the sampled luminosity range, with no attempt made to extrapolate toward the faint or bright luminosity ends using the evolved local LF. This eliminates any assumption, and yields robust lower limits purely obtained from the data. The dependence of the derived SFRD on the faint/bright end extrapolation is illustrated by the (solid and dashed) curves in Figure 4, which were obtained using the average pure luminosity evolution of the two local LFs derived in Section 2.5, and integrating over the entire SFRD curve. Hence, the largest uncertainty in the derivation of the SFRD based on radio data arises from the uncertainty in the shape of the local radio LF (assumed to not change with redshift) and the associated extrapolation below the faintest luminosity sampled by the data. 


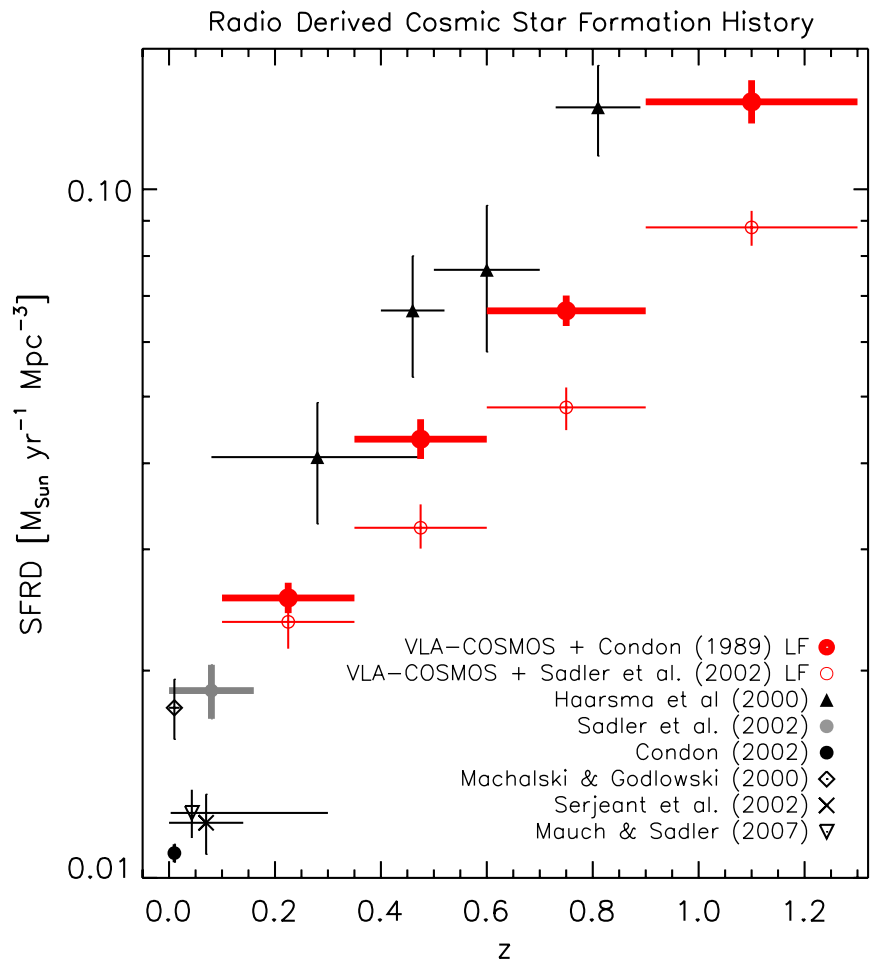

Figure 5. The comparison of radio-derived CSFHs. The different samples are indicated in the panel. The bold, filled red circles show the VLA-COSMOS results, when the Condon (1989) local LF is used; the open red circles show the results when the Sadler et al. (2002) local LF is used. The results based on other radio-based studies are also shown, and are indicated in the panel.

Table 2

Star-Formation Rate Density ${ }^{\mathrm{a}}$

\begin{tabular}{lcc}
\hline \hline Redshift & \multicolumn{2}{c}{ SFRD $\left(M_{\odot} \mathrm{yr}^{-1}\right)$} \\
\cline { 2 - 3 } Range & Condon $(1989) \mathrm{LF}$ & Sadler et al. $(2002) \mathrm{LF}$ \\
\hline $0.1<z \leqslant 0.35$ & $0.025_{-0.001}^{+0.001}$ & $0.023_{-0.002}^{+0.002}$ \\
$0.35<z \leqslant 0.6$ & $0.043_{-0.003}^{+0.003}$ & $0.032_{-0.002}^{+0.003}$ \\
$0.6<z \leqslant 0.9$ & $0.067_{-0.003}^{+0.003}$ & $0.048_{-0.004}^{+0.003}$ \\
$0.9<z \leqslant 1.3$ & $0.134_{-0.009}^{+0.010}$ & $0.088_{-0.005}^{+0.005}$ \\
\hline
\end{tabular}

Note. ${ }^{a}$ Derived Using the Calibration Given in Bell (2003, see Equation (5)) from 1.4 GHz VLA-COSMOS Data $\left(H_{0}=70, \Omega_{M}=0.3, \Omega_{\Lambda}=0.7\right)$.

We compare our SFRD results (given in Table 2), obtained by integrating over the best-fit evolved local LF in each redshift range (see curves in Figure 3), with other radio-based estimates in Figure 5. The expected steep decline in the SFRD since $z \sim 1$ is reproduced by our data. Our results are consistent within the errors with the results from Haarsma et al. (2000, who used the Condon 1989 local LF), although their results are on average higher. Note also that our statistical CSFH uncertainties (bold red crosses in Figure 5) are significantly smaller compared with the Haarsma et al. (2000) results, as a result of the larger sample utilized here. Our results are also qualitatively consistent with those obtained by Ivison et al. (2007), based on radio image stacking of UV-selected galaxies in the AEGIS20 $0^{13}$ survey.

Seymour et al. (2008) have used VLA/MERLIN radio frequency observations of the $13^{\mathrm{H}}$ XMM-Newton/Chandra Deep

\footnotetext{
13 The AEGIS20 survey is a radio survey that is part of the larger All-wavelength Extended Groth Strip International Survey (AEGIS), conducted by NRAO's VLA.
}

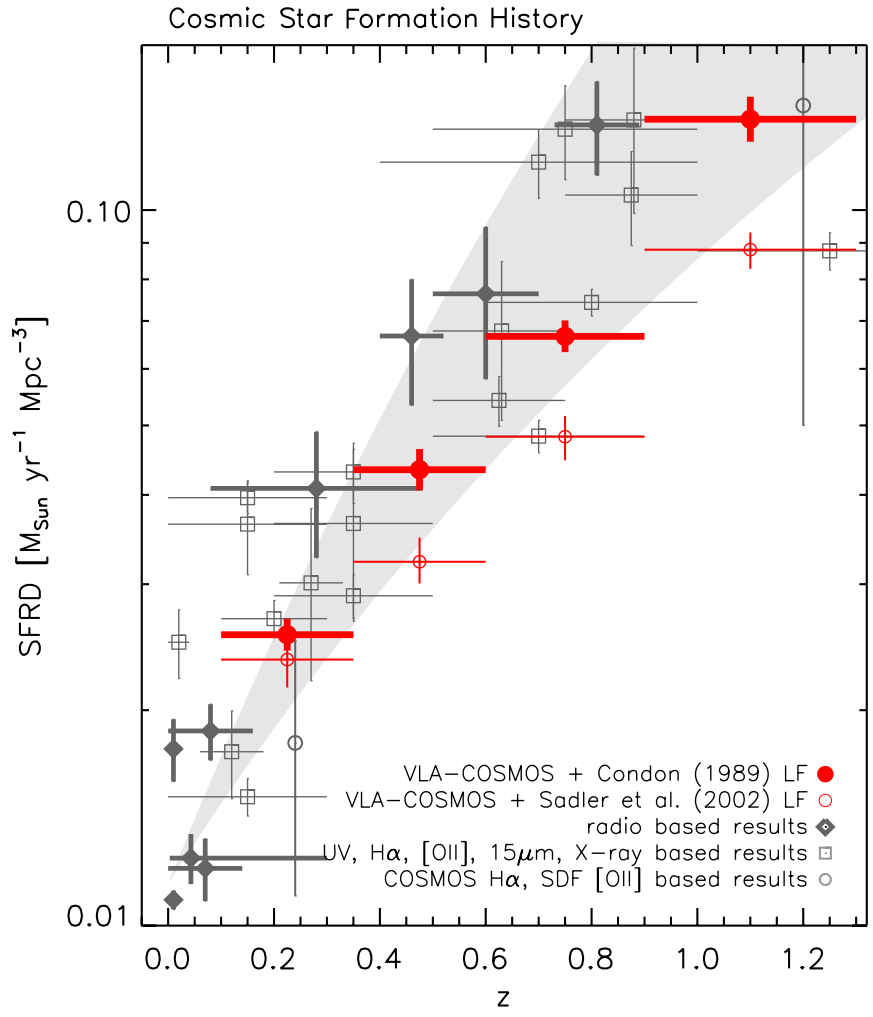

Figure 6. The comparison of the VLA-COSMOS derived CSFH (red symbols; see Figure 5 for details) with other wavelength-based results. The compilation of CSFHs contains UV-, $\mathrm{H} \alpha$-, FIR-, and X-ray-based results, corrected for dust obscuration using luminosity-dependent corrections (open gray squares; see Hopkins 2004 for details). The thick gray diamonds denote local radio estimates (Machalski \& Godlowski 2000; Condon et al. 2002; Sadler et al. 2002; Serjeant et al. 2002). For all radio data the $1.4 \mathrm{GHz}$ luminosity to SFR calibration given in Bell (2003, see Equation (5)) here is used. The gray-shaded area shows the CSFH derived by LF05, based on $24 \mu \mathrm{m}$ data. The open gray circles denote (1) the $\mathrm{H} \alpha$ derived SFRD at $z=0.24$ in the COSMOS field, corrected for both dust-obscuration and AGN contribution (Shioya et al. 2008), and (2) the dust-obscuration corrected SFRD at $z=1.2$ derived using [O II] emission galaxies in the Subaru Deep Field (SDF; see Takahashi et al. 2007 for details).

Field to derive the CSFH. Their findings are qualitatively consistent with those from Haarsma et al. (2000, shown in Figure 5) when they use the Mauch \& Sadler (2007) local LF evolved in luminosity with an a priori set value of $\alpha_{L}=2.5$ (note that this local LF has a lower normalization than Sadler et al. 2002; see the local results in Figures 4 and 5). Further, they used a different radio luminosity to SFR relation that is consistent with 0.84 times the Bell (2003) calibration used here (see Seymour et al. 2008 for details). Hence, this implies that their results are significantly higher than the VLA-COSMOS results. Further, given the differences between the local radio LFs outlined in Section 2.4.1, if Seymour et al. (2008) had chosen to use the Condon (1989) local LF with otherwise the same assumptions, they would have obtained even higher SFRD values. We believe that the main reason for the differences between our results and those by Seymour et al. (2008) is likely a combination of (1) their a priori assumed evolution of the local LF, contrary to constraining the evolution by their data and (2) the inclusion of a fraction of lower power (i.e., radio quiet) AGNs in their SF galaxy sample, while our sample may oversubtract composite objects (see also Section 4).

In Figure 6 we compare the VLA-COSMOS derived CSFH data with results from previous studies based on a range of SF estimators-UV, optical, FIR, total IR, and radio. A luminosity- 
dependent obscuration correction was used where necessary (see Hopkins 2004, and references therein). Overall, our derived $\mathrm{CSFH}$ agrees with the general trend of a rapid decline by almost an order of magnitude in the cosmic SFRD since $z \sim 1$. A possibly slower decline is suggested by our data if the Sadler et al. (2002) LF is used. However, as the uncertainties due to the faint-end extrapolation are significant, no robust conclusions can be made at this point.

\subsection{The CSFH of Intensely SF Galaxies}

The VLA-COSMOS SF galaxy sample constrains well the high end of the LF for SF galaxies. Given the $2 \mathrm{deg}^{2}$ VLA-COSMOS field, the comoving volume sampled up to $z=1.3$ is $\sim 1.3 \times 10^{7} \mathrm{Mpc}^{3}$, corresponding roughly to the volume observed locally by SDSS-DR1. Thus, for the first time this allows a robust derivation of the CSFH for galaxies forming stars at rates of $\gtrsim 100 M_{\odot} \mathrm{yr}^{-1}$ out to $z=1.3$. Such radioselected galaxies are equivalent to the ultra-luminous IR galaxies (ULIRGs, $L_{\mathrm{IR}}>10^{12} L_{\odot}$ ), and it is noteworthy that the VLA-COSMOS survey is sensitive to a complete sample of these galaxies out to $z \sim 1$ (see Figure 16 in S08).

In order to derive the evolution of the SFRD at the highluminosity end, we integrate the SFRD curve (obtained from the best-fit pure radio luminosity evolution in each redshift bin, see Figure 3) only for our SF galaxies that have $L_{1.4} \gtrsim 2.34 \times$ $10^{23} \mathrm{~W} \mathrm{~Hz}^{-1}$, which corresponds to $L_{\mathrm{IR}}>10^{12} \widetilde{L}_{\odot}$ given the adopted total IR-radio correlation (Bell 2003). For this we use the local LF given by Sadler et al. (2002), as it appears to be better suited for the high-luminosity end compared with the Condon (1989) LF (see Figure 2). Note that for these highly luminous galaxies the extrapolation uncertainties are not as significant as for the overall SFRD, as this sample is almost complete in all three high redshift ranges. A small extrapolation to the faint end, given the form of the evolved local LF, is necessary only in the last redshift bin. For a consistent comparison between our radio and IR (LF05) results we convert the total IR luminosity to SFRs consistently using the calibration given by Bell (2003). The results are shown in Figure 7. The evolution of our SF ULIRGs is consistent with the lower envelope derived by LF05. However, it is marginally flatter, suggesting a slower evolution of SF ULIRGs since $z=1.3$. This will be further discussed in the next section.

\section{DISCUSSION}

\subsection{The Evolution of the Most Intensely Star-Forming Galaxies}

Making use of our large statistical sample of radio-selected SF ULIRGs complete out to $z \sim 1$, we have derived the CSFH of the most intensely SF galaxies ( $\gtrsim 100 M_{\odot} \mathrm{yr}^{-1}$ ) out to $z=1.3$. Our evolution of the cosmic SFR in SF ULIRGs qualitatively agrees with previous MIR-based results (LF05; see Figure 7). However, we find a slightly slower evolution than that predicted by the MIR results. The major cause for this difference is currently unclear, nonetheless there are likely three effects that may contribute.

1. No attempt has been made to minimize the AGN contamination in the $24 \mu \mathrm{m}$-selected sample, possibly causing an overestimate in the MIR-derived SFRD evolution for ULIRGs. For example, Caputi et al. (2007) have found more than $10 \%$ of $24 \mu \mathrm{m}-\mathrm{AGN}$ at $z \sim 1$, and a factor of 2 more at $z \sim 2$, suggesting that the AGN fraction in MIR-selected samples increases with redshift. The AGN

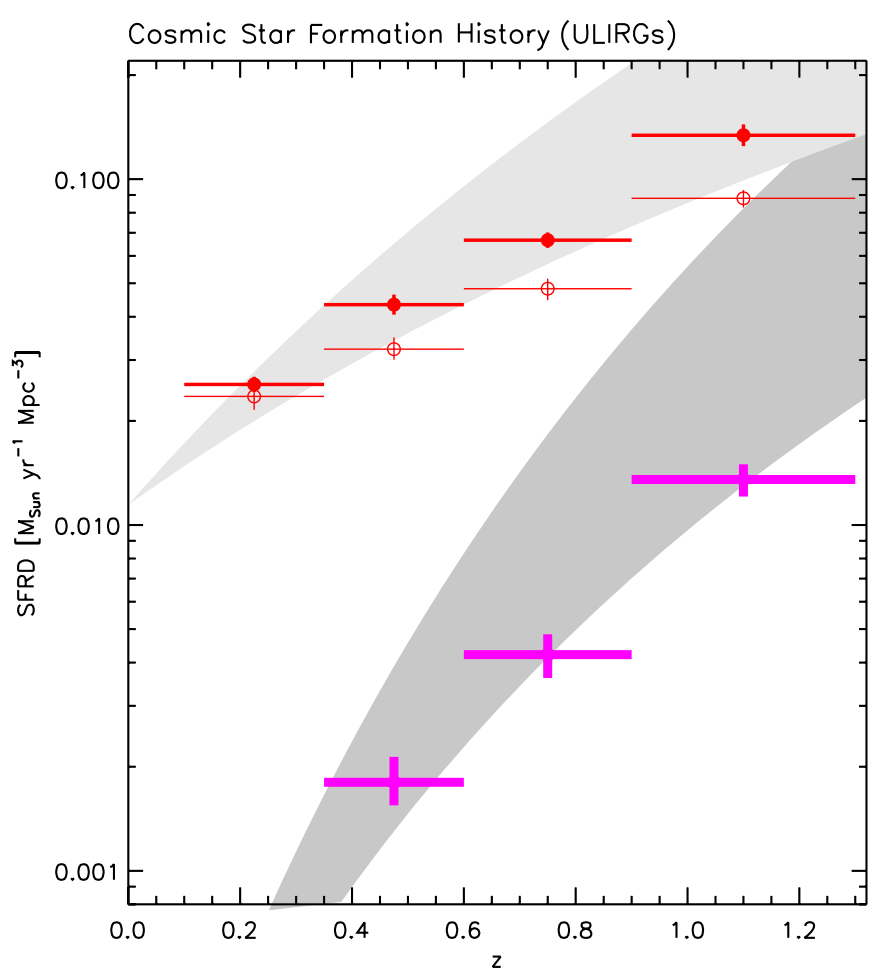

Figure 7. The CSFH derived from VLA-COSMOS data for the overall population (red symbols as in Figure 6), and only for SF ULIRGs (magenta symbols, derived using the Sadler et al. 2002 local LF, evolved in luminosity, in each redshift bin). The CSFH for the entire population (light-gray-shaded curve) and the ULIRG population (dark-gray-shaded curve) derived from the evolved total IR LF (LF05) are also shown. The IR- and radio-based SFRs have been put on the same relative scale (see the text for details). Note that the VLA-COSMOS data suggest a slightly slower evolution of the SF ULIRG population with redshift.

fraction in MIR samples may also be a function of stellar mass. Although, at higher redshift than that analyzed here (implying a different cosmological era), Daddi et al. (2007) have demonstrated that at $z \sim 2$ the MIR-AGN fraction is indeed a function of stellar mass, and reaches $\sim 50-60 \%$ for masses greater than $4 \times 10^{10} M_{\odot}$. The median stellar mass of the VLA-COSMOS SF galaxies (obtained via SED fitting; see S08 for details) is $\sim 7 \times 10^{10} M_{\odot}$.

2. Particular care was taken to separate the VLA-COSMOS population into SF and AGN galaxies. Nonetheless, it has to be noted that some uncertainty, due to AGN contamination as well as incompleteness of the SF galaxy sample, exists, especially on the galaxy-by-galaxy and composite (SF plus AGN) galaxy level (see S08 for details). It is also worth noting that two significant large-scale structure components exist in our highest-redshift bin (see e.g., Scoville et al. 2007a) that may affect the fraction of SF and AGN galaxies present in this particular redshift range.

3. The local IRAS total IR and the Sadler et al. (2002) radio LFs, used for the derivation of these results, are not perfectly similar. There may also be a volume density excess of SF galaxies with high-radio luminosities at $z>0.6$, compared with the Sadler et al. LF evolved only in luminosity. In addition, the total IR-radio correlation, used to select ULIRGs from our radio sample, carries its own uncertainty and intrinsic astrophysical scatter (Bressan et al. 2002; Bell 2003). Therefore, it is not immediately obvious whether the same results would be expected based on both-radio and MIR — star-formation indicators. 
In summary, it is encouraging that the same qualitative behavior of the evolution of the ULIRG population is observed with the two independent (radio and MIR) SFR indicators. However, further dedicated studies of the details will prove most interesting in understanding the quantitative differences seen in Figure 7.

\subsection{The Rapid Decline of the CSFH Since $z \sim 1$}

Our overall CSFH (Figure 6) agrees well with past findings when these are corrected for dust-obscuration as needed. This verifies the assumptions about large-dust-obscuration corrections required, especially for short-wavelength (e.g., UV) starformation tracers. Our radio data independently confirm the $\sim 1$ order of magnitude decline in the cosmic SFR since $z \sim 1$.

Based on UV- and IR-based SFR/morphology studies (Wolf et al. 2005; Bell et al. 2005; Melbourne et al. 2005; Hammer et al. 2005; Zamojski et al. 2007), this rapid decline in the overall CSFH is expected to be driven by normal spiral galaxies. For example, Bell et al. (2005) have performed a detailed morphological study of a galaxy sample $(z=0.7)$ with SFRs $\gtrsim 10 M_{\odot} \mathrm{yr}^{-1}$. They have demonstrated that physical processes that do not substantially affect galaxy morphology, such as minor mergers, gas consumption, and weak interactions with satellite galaxies, may be most important for the rapid decline in the overall CSFH (see also e.g., Somerville et al. 2001).

The sample analyzed here, however, is most sensitive to ULIRGs (SFR $\gtrsim 100 M_{\odot} \mathrm{yr}^{-1}$ ), and those are the systems that are a priori expected to be a reflection of galaxy merging (based on local ULIRG morphology studies; Sanders \& Mirabel 1996). This implies that the rapid decline in CSFH of our ULIRGs (Figure 7) may be more affected by galaxy mergers than the overall CSFH decline since $z \sim 1$. Intriguingly, our derived LF evolution is very similar to the evolution of the galaxy closepair fraction, derived for bright galaxies ( $>L_{V}^{*}$; Kartaltepe et al. 2007 ) in the COSMOS field, well described with a power law with an index of $3.1 \pm 0.1$ (or $2.2 \pm 0.1$ when pure luminosity evolution is considered; see also e.g., Lotz et al. 2008). This strong evolution of the bright close-pair fraction, combined with a similarly strong evolution of the CSFH of ULIRGs derived here suggests that major mergers may play an important role in the rapid decline of the CSFH since $z \sim 1$ for the most intensely SF galaxies. Thus, the observed steep evolution of our ULIRG population may be a good record of merger rate evolution, combined with gas content evolution.

\section{SUMMARY}

We have derived the CSFH out to $z=1.3$ using the largest sample to date of radio-selected SF galaxies observed at $1.4 \mathrm{GHz}(20 \mathrm{~cm})$ in the VLA-COSMOS survey. The large increase in the number of radio-selected SF galaxies out to high redshift, compared with previous studies, allowed us to constrain the evolution of the $1.4 \mathrm{GHz}$ LF well for radioselected $S F$ galaxies, as well as to significantly reduce the statistical uncertainties of the radio-derived CSFH. We find that the uncertainties are ruled by the differences in the shape of the local radio LFs present in the literature. A pure radio luminosity evolution of VLA-COSMOS SF galaxies is well described with $L_{*} \propto(1+z)^{2.1 \pm 0.2}$, when evolving the Sadler et al. (2002) local $\mathrm{LF}$, or with $L_{*} \propto(1+z)^{2.5 \pm 0.1}$ when evolving the Condon (1989) local LF. Although encompassing a relatively broad range, both values are consistent with previously derived evolution of SF galaxies (e.g., Condon et al. 2002; Hopkins 2004; Cowie et al. 2004; Afonso et al. 2005).

Our overall CSFH agrees well with past findings when these are corrected for dust obscuration where needed. This verifies the assumptions about large-dust-obscuration corrections required, especially for short-wavelength (e.g., UV) starformation tracers. Making use of our large statistical sample of radio-selected SF ULIRGs complete out to $z \sim 1$, we have robustly constrained the high end of the SF galaxy LF at different cosmic times. Using these, we have derived the CSFH of the most intensely SF galaxies ( $\gtrsim 100 M_{\odot} \mathrm{yr}^{-1}$; i.e., SF ULIRGs) out to $z=1.3$. We find, on average, a slower evolution of the cosmic SFR in SF ULIRGs than that predicted by MIR results. This is consistent with the fraction of SF galaxies in MIR samples likely becoming lower with increasing redshift and/or stellar mass.

C.C. acknowledges support from the Max-Planck Society and the Alexander von Humboldt Foundation through the Max-Planck-Forschungspreis 2005. G.Z. acknowledges support from two contracts PRIN-INAF 2005 and 2007. T.P. acknowledges support from PSC-CUNY grant \# 69612-00 38.

\section{REFERENCES}

Afonso, J., Georgakakis, A., Almeida, C., Hopkins, A. M., Cram, L. E., Mobasher, B., \& Sullivan, M. 2005, ApJ, 624, 135

Barger, A. J., Cowie, L. L., \& Wang, W.-H. 2007, ApJ, 654, 764

Bell, E. F. 2003, ApJ, 586, 794

Bell, E. F., Zheng, X. Z., Papovich, C., Borch, A., Wolf, C., \& Meisenheimer, K. 2007, ApJ, 663, 834

Bell, E. F., et al. 2005, ApJ, 625, 23

Best, P. N., et al. 2005, MNRAS, 362, 9

Bondi, M., Ciliegi, P., Schinnerer, E., Smolčić, V., Jahnke, K., Carilli, C., \& Zamorani, G. 2008, ApJ, 681, 1129

Bressan, A., Silva, L., \& Granato, G. L. 2002, A\&A, 392, 377

Caputi, K. I., et al. 2007, ApJ, 660, 97

Condon, J. J. 1987, ApJS, 65, 485

Condon, J. J. 1989, ApJ, 338, 13

Condon, J. J. 1992, ARA\&A, 30, 575

Condon, J. J., Cotton, W. D., \& Broderick, J. J. 2002, AJ, 124, 675

Cooper, M. C., et al. 2008, MNRAS, 383, 1058

Cowie, L. L., Barger, A. J., Fomalont, E. B., \& Capak, P. 2004, ApJ, 603, L69

Cucciati, O., et al. 2006, A\&A, 458, 39

Daddi, E., et al. 2007, ApJ, 670, 173

Fomalont, E. B., Windhorst, R. A., Kristian, J. A., \& Kellerman, K. I. 1991, AJ, 102,1258

Haarsma, D. B., et al. 2000, ApJ, 544, 641

Hammer, F., Crampton, D., Lilly, S. J., Le Fevre, O., \& Kenet, T. 1995, MNRAS, 276, 1085

Hammer, F., Flores, H., Elbaz, D., Zheng, X. Z., Liang, Y. C., \& Cesarsky, C. 2005, A\&A, 430, 115

Hopkins, A. M. 2004, ApJ, 615, 209

Hopkins, A. M., \& Beacom, J. F. 2006, ApJ, 651, 142

Huynh, M. T., Jackson, C. A., Norris, R. P., \& Fernandez-Soto, A. 2008, AJ, 135,2470

Ivison, R. J., et al. 2007, ApJ, 660, L77

Kartaltepe, J. S., et al. 2007, ApJS, 172, 320

Le Floc'h, E., et al. 2005, ApJ, 632, 169

Lotz, J. M., et al. 2008, ApJ, 672, 177

Machalski, J., \& Godlowski, W. 2000, A\&A, 360, 463

Mauch, T., \& Sadler, E. M. 2007, MNRAS, 375, 931

McCracken, H. J., et al. 2007, ApJS, 172, 314

Melbourne, J., Koo, D. C., \& Le Floc'h, E. 2005, ApJ, 632, L65

Richards, E. A., Kellermann, K. I., Fomalont, E. B., Windhorst, R. A., \& Partridge, R. B. 1998, AJ, 116, 1039

Sadler, E. M., et al. 2002, MNRAS, 329, 227

Sandage, A., Tammann, G. A., \& Yahil, A. 1979, ApJ, 232, 352

Sanders, D. B., \& Mirabel, I. F. 1996, ARA\&A, 34, 749

Schinnerer, E., et al. 2007, ApJS, 172, 46

Schmidt, M. 1968, ApJ, 151, 393 
Scoville, N., et al. 2007a, ApJS, 172, 1

Scoville, N., et al. 2007b, ApJS, 172, 150

Serjeant, S., Gruppioni, C., \& Oliver, S. 2002, MNRAS, 330, 62

Seymour, N., et al. 2008, MNRAS, 386, 1695

Shioya, Y., et al. 2008, ApJS, 175, 128

Smolčić, V., et al. 2006, MNRAS, 371, 121

Smolčić, V., et al. 2008, ApJS, 177, 14

Somerville, R. S., Primack, J. R., \& Faber, S. M. 2001, MNRAS, 320,504
Takahashi, M. I., et al. 2007, ApJS, 172, 456

Windhorst, R. A., Fomalont, E. B., Kellermann, K. I., Partridge, R. B., Richards, E., Franklin, B. E., Pascarelle, S. M., \& Griffiths, R. E. 1995, Nature, 375, 471

Wolf, C., et al. 2005, ApJ, 630, 771

Zamojski, M. A., et al. 2007, ApJS, 172, 468

Zheng, X. Z., Bell, E. F., Papovich, C., Wolf, C., Meisenheimer, K., Rix, H.-W., Rieke, G. H., \& Somerville, R. 2007, ApJ, 661, L41

Zheng, X. Z., et al. 2006, ApJ, 640, 784 